\title{
The effects of four days of continuous striatal microdialysis on indices of dopamine and serotonin neurotransmission in rats
}

\author{
Terry E. Robinson ${ }^{1,2}$ and Dianne M. Camp ${ }^{1}$ \\ ${ }^{1}$ Department of Psychology and ${ }^{2}$ Neuroscience Program, University of Michigan, \\ Ann Arbor, MI 48109 (U.S.A.)
}

(Received 8 July 1991)

(Revised version received 10 September 1991)

(Accepted 20 September 1991)

Key words: Dialysis; Caudate nucleus; Amphetamine; Cocaine; Methods

The effects of 4 days of continuous microdialysis with a small-diameter concentric-style probe on indices of striatal dopamine (DA) and serotonin neurotransmission were assessed. It was found that over 4 days of dialysis, there was a marked time-dependent decrease in the basal concentrations of 3,4-dihydroxyphenylacetic acid (DOPAC) and homovanillic acid (HVA) in dialysate and in amphetamine-stimulated DA release. In contrast, there was no decrease in basal DA or in the ability of cocaine to elevate the concentration of DA in dialysate over the same period of time. There were only very modest changes in dialysate levels of the serotonin metabolite, 5-hydroxyindoleacetic acid (5-HIAA), relative to the marked changes in DA metabolites. It is suggested that 4 days of continuous dialysis does not result in a non-specific decrease in diffusibility of these compounds into the dialysis probe, but that the changes are more likely due to probe-induced damage to the nigrostriatal DA system. It is also suggested that a "stable" basal concentration of DA in dialysate is an especially poor indicator of the integrity of the dopaminergic input to the striatum. The implications of these findings for within-subjects design microdialysis experiments are discussed.

\section{Introduction}

Intracerebral microdialysis has rapidly become a popular method for in vivo sampling of neurotransmitters and their metabolites in neural tissue of awake, moving animals (Ungerstedt and Hallström, 1987). The technique is relatively new, however, and there have been few studies designed to characterize the effects of repeated sampling over extended periods of time, either with a microdialysis probe left in place (Imperato and Di Chiara, 1984, 1985; Korf and Venema, 1985; Westerink and Tuinte, 1986; Reiriz et al.,

Correspondence: Dr. Terry E. Robinson, Neuroscience Building, University of Michigan, 1103 East Huron Street, Ann Arbor, MI 48109, U.S.A. Tel.: (313) 763-4361. Fax: (313) 936-2690. E-Mail: GB92@UMichUM.BitNet.
1989), or with multiple insertions of a probe at different points in time (Camp and Robinson, 1991). Initial attempts at continuous dialysis with a chronically implanted dialysis probe in the nigrostriatal dopamine (DA) system were not very encouraging. For example, Westerink and Tuinte (1986) reported that between $24 \mathrm{~h}$ and 7 days after probe implantation the basal concentrations of striatal DA and 3,4-dihydroxyphenylacetic acid (DOPAC) in dialysate progressively declined, as did the magnitude of $\mathrm{K}^{+}$- and amphetaminestimulated DA release. This decline was attributed to cellular reactions at the probe tip, including gliosis, which were thought to impede the diffusion of neurochemicals from the extracellular fluid into the dialysis membrane, and vice versa (Hamberger et al., 1983; Imperato and $\mathrm{Di}$ Chiara, 1985; Westerink and Tuinte, 1986; Benveniste and Diemer, 1987). 
However, in some of the previous studies involving continuous dialysis large diameter Ushaped ("loop" style) probes were used (Westerink and Tuinte, 1986; Reiriz et al., 1989), and these can cause considerable non-specific neural damage. Different style probes have been reported to have different effects on the stability of basal DA in dialysate over 2 days, the effect of tetrodotoxin (TTX) and low $\mathrm{Ca}^{2+}$ conditions on DA release, and the disruption of blood flow and glucose utilization seen in locations distant from the site of probe implantation (Westerink and de Vries, 1988; Benveniste and Hüttemeier, 1990; Santiago and Westerink, 1990). Therefore, in exploring the feasibility of within-subjects design microdialysis experiments, we thought it was important also to characterize the effects of daily sampling from a chronically implanted, small-diameter, concentric-style probe. This type of probe causes much less non-specific damage than a U-shaped probe, and a unilateral placement avoids the symmetrical bilateral damage produced by a transverse probe. The effects of 4 days of continuous dialysis on both basal and stimulated DA release were assessed.

\section{Methods}

\section{Subjects}

The subjects were adult female Holtzman rats weighing $250-350 \mathrm{~g}$. Animals were housed individually in wire, hanging cages with free access to food and water, in a light and temperature controlled room (lights on at 08:00 and off at 18:00 h).

\section{Protocol \\ Experiment 1. Continuous dialysis over 4 days: effects of repeated amphetamine treatment. Each animal was anesthetized with sodium pentobarbi- tal, supplemented with methoxyflurane, and a single concentric-style microdialysis probe was stereotaxically lowered into the corpus of the neostriatum. The dialysis probe had a $4.0-\mathrm{mm}$ - long dialysis surface with an outer diameter of $250 \mu \mathrm{m}$, and has been described in detail else- where (Robinson and Whishaw, 1988; Robinson}

and Camp, 1991). The dialysis probe was permanently fixed in place with skull screws and dental acrylic. After the dental acrylic had cured, the animal was transferred to a test chamber, and the inlet tubing was attached to a liquid swivel (Instec) mounted on the end of a counter-balanced arm centered over the test chamber. The animal was connected to the swivel via a flexible tether constructed from model airplane control cable, and this was attached to an elastic harness 1ocated around the animal's torso. The animals were then left overnight, and the perfusion solution was pumped continuously through the probe at $0.3 \mu \mathrm{l} / \mathrm{min}$. The perfusion solution contained $147 \mathrm{mM} \mathrm{Na}{ }^{+}, 4.0 \mathrm{mM} \mathrm{K}{ }^{+}, 2.3 \mathrm{mM} \mathrm{Ca}^{2+}$ and $155.6 \mathrm{mM} \mathrm{Cl}^{-}, \mathrm{pH} 6.0$.

The next day (defined here as day 1) the syringe pump was set at $1.5 \mu \mathrm{l} / \mathrm{min}$ and $30-60$ min later at least 3 baseline samples of dialysate were collected over 20-min intervals. After this, half the animals received an i.p. injection of 2.0 $\mathrm{mg} / \mathrm{kg}$ of D-amphetamine sulfate (weight of the salt), and half an injection of an equal volume of $0.9 \%$ saline. Sample collection then continued for $200 \mathrm{~min}$. The pump was then turned down to 0.3 $\mu \mathrm{l} / \mathrm{min}$ until the next day (day 2), when the entire procedure was repeated. This continued for 4 days, except on day 3 only basal values were quantified. On day 4 all animals were given 2.0 $\mathrm{mg} / \mathrm{kg}$ of amphetamine (after basal samples were collected), including those animals that previously received saline. The animals were tested in pairs, consisting of 1 saline and 1 amphetamine pretreated rat.

At the completion of each experiment the dialysis tubing was cut close to the dental acrylic "cap", the open ends sealed with epoxy, and the animals returned to their home cage. A few days later the animals received an overdose of sodium pentobarbital and were perfused through the heart with saline followed by $10 \%$ formalin, with the dialysis probe in place. The brains were cut frozen, stained with cresyl violet, and the sections examined to determine the exact location of the dialysis probe.

Experiment 2. Continuous dialysis over 4 days: effects of repeated cocaine. The protocol for this experiment was exactly as described for Exp. 1, 
except for the following modifications. (1) The dialysis probe was lowered via a chronically implanted guide cannula, which was placed stereotaxically above the striatum at least 2 weeks prior to a test session (Robinson and Camp, 1991). When the dialysis probe was lowered into the striatum via the guide cannula, the day before a test session, the animals were only briefly anesthetized with methoxyflurane. (2) On days 1-3 half the animals received $15 \mathrm{mg} / \mathrm{kg}$ of cocaine $\mathrm{HCl}$ (i.p., free base) instead of amphetamine, and on day 4 all the animals received $15 \mathrm{mg} / \mathrm{kg}$ of cocaine. (3) The perfusion solution consisted of $145 \mathrm{mM} \mathrm{NaCl}, 2.7 \mathrm{mM} \mathrm{KCl}, 1.2 \mathrm{mM} \mathrm{CaCl}_{2}, 1.0$ $\mathrm{mM} \mathrm{MgCl}_{2}$ and $0.2 \mathrm{mM}$ ascorbic acid, $\mathrm{pH} 7.3$ (Moghaddam and Bunney, 1989).

\section{Assay of dialysate and data analysis}

Dialysate samples were assayed within $20-40$ min after collection using high performance liquid chromatography with electrochemical detection, as described in detail elsewhere (Robinson and Camp, 1990, 1991).

Data are included only for those animals in which the probe was located in the corpus of the neostriatum and for which data were obtained on all 4 days of testing. The concentration of each compound is expressed in $\mathrm{fmol} / \mathrm{min}$, and all values were corrected for probe recovery (calculated in vitro at $37^{\circ} \mathrm{C}$ ). Note that in vitro recovery procedures do not provide an accurate estimate of the actual interstitial concentration of DA (Benveniste and Hansen, 1991; Morrison et al., 1991) but were used here primarily to minimize experimental error due to variation in recovery, attributable to variation in probe construction.

Statistical analyses were conducted using a Macintosh II computer and Systat $5.0^{\circledR}$ or StatView II ${ }^{\circledR}$ software. The effect of days of dialysis for the drug-treated groups (amphetamine and cocaine) was determined by application of a within-subjects design analysis of variance for each compound. Comparisons between the saline and drug pretreated groups (on day 4) were made using a between-within analysis of variance for each compound. If the outcome of the analysis of variance warranted, pairwise comparisons were made using either the Fisher's Least Significant
Difference (LSD) test or independent $t$ tests (2-tailed probabilities).

\section{Results}

\section{Experiment 1}

Fig. 1 shows the average basal concentrations of DA, DOPAC, homovanillic acid (HVA) and 5-hydroxyindoleacetic acid (5-HIAA) in dialysate on each of the 4 days of testing in Exp. 1. There was no difference between amphetamine $(n=6)$ and saline pretreated rats $(n=5)$ in the basal concentrations of DA $(F=1.05, P=0.33)$, DOPAC $(F=0.36, P=0.56)$, HVA $(F=0.71, P$ $=0.42)$ or 5 -HIAA $(F=0.02, P=0.90)$ across the 4 days of testing (2-way ANOVAs) and, therefore, these groups were pooled to simplify data presentation and analysis in Fig. 1.

Fig. 1 shows that in Exp. 1 there was a marked decrease in the basal concentrations of DOPAC and HVA in dialysate across the 4 days of testing (for DOPAC, $F=20.58, P<0.0001$; for HVA, $F=21.54, P<0.0001)$. There was a large de-

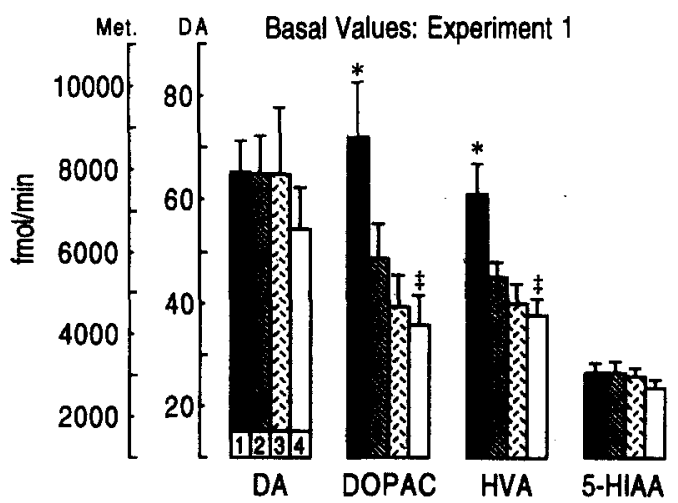

Fig. 1. The mean (+SEM) basal concentrations of dopamine (DA), 3,4-dihydroxyphenylacetic acid (DOPAC), homovanillic acid (HVA) and 5-hydroxyindoleacetic acid (5-HIAA) in dialysate on each of 4 consecutive days of continuous dialysis in Exp. 1 (each day is represented by the columns numbered $1-4$, for each compound). Note that there is a different vertical axis for DA and the 3 monoamine metabolites (Met). For each compound: ${ }^{*}$, indicates day 1 values that differed significantly from all other days; $\ddagger$, indicates day 4 values that differed from day 2 values; ( $n=11$; statistics based on withinsubjects ANOVAs followed by pairwise comparisons with Fisher's LSD tests where appropriate; see text). 
crease in both of the DA metabolites between days 1 and $2(P<0.05$, Fisher's LSD test $)$, and a further significant decrease between days 2 and 4
$(P<0.05)$. The day 3 value was intermediate between days 2 and 4 (Fig. 1). In contrast, there was no significant change in basal DA across the

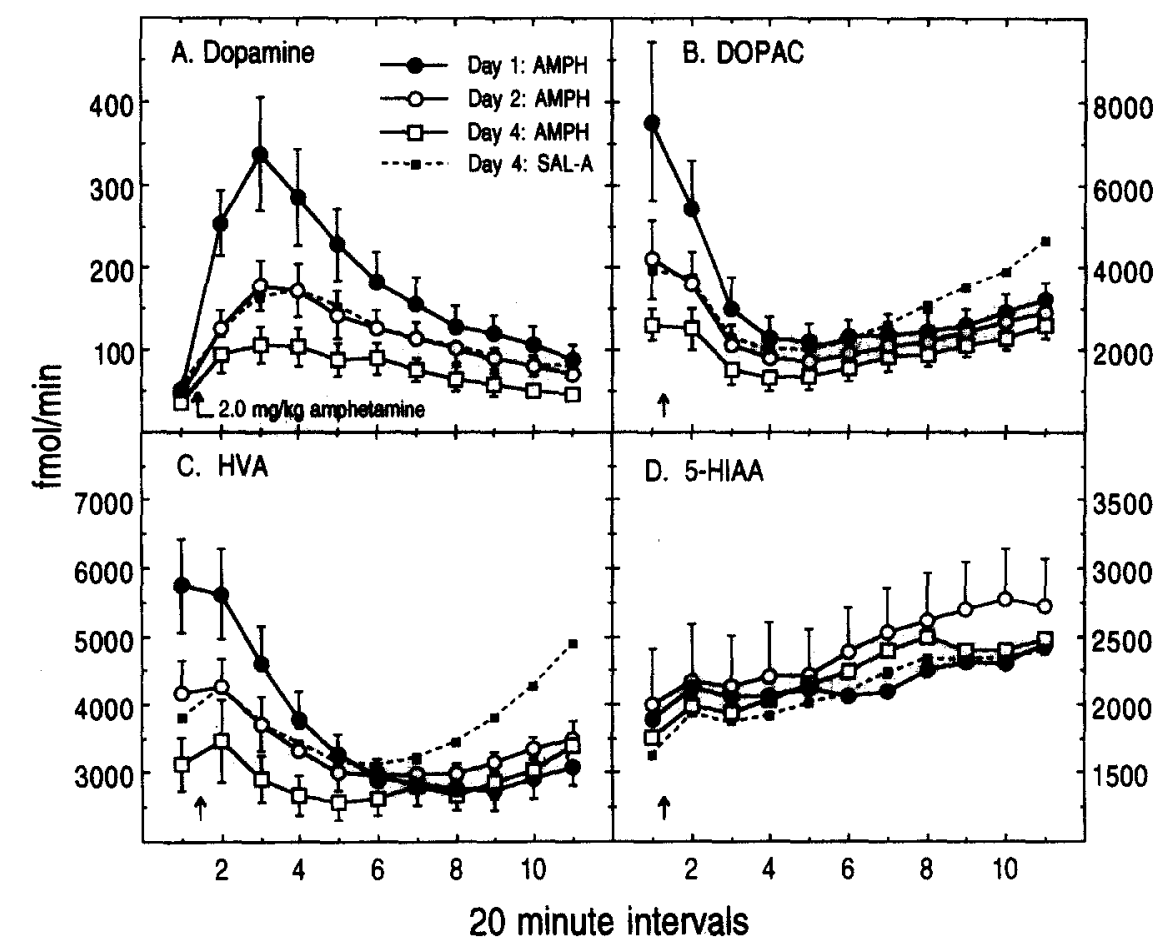

Fig. 2. The effect of $2.0 \mathrm{mg} / \mathrm{kg}$ of D-amphetamine sulfate on the mean ( $\pm \mathrm{SEM}$ ) concentration of DA (panel A), DOPAC (panel B), HVA (panel C) and 5-HIAA (panel D) in dialysate on days 1, 2 and 4 after probe implantation ("AMPH" groups). The "day 4: SAL-A" group represents animals that received saline on the first 3 days of dialysis (data not shown) and their response to amphetamine on day 4 of dialysis is illustrated here. The first $20 \mathrm{~min}$ interval represents the basal concentration of each compound (mean of 3 determinations), and each subsequent $20 \mathrm{~min}$ interval represents successive samples obtained beginning immediately after the injection of amphetamine (given when indicated by the arrow in each panel). Dopamine: the effect of amphetamine on DA was significantly decreased on days 2 and 4, relative to day 1 ; and the day 4 values were significantly lower than the day 2 values (2-way ANOVAs with repeated measures on both factors, day X interval; see text). Two-way ANOVAs with only 1 repeated measure (interval) were used to compare the AMPH and saline pretreated groups. When saline pretreated animals received amphetamine on the 4th day of dialysis they showed a significantly smaller increase in DA than did the "day 1: AMPH" group, but did not differ from either the "day 2: AMPH" or the "day 4: AMPH" groups. That is, the magnitude of the decrease in amphetamine-stimulated DA release (relative to "day 1: AMPH") was just as great in saline pretreated animals as in animals that received amphetamine on all days of testing. DOPAC: amphetamine produced a decrease in the concentration of DOPAC in dialysate on all days of dialysis (the main effect of interval was significant for all comparisons). An overall 2-way ANOVA with repeated measures on both factors (day $\mathrm{X}$ interval) was significant $\left(F_{\mathrm{d}}=12.86, P=0.002, F_{\mathrm{i}}=10.98, P<0.0001, F_{\mathrm{interaction}}=6.38\right.$, $P<0.0001$ ). As expected by the decrease in basal DOPAC, subsequent 2-way ANOVAs comparing each day showed that there were significant differences between day 1 vs. day $2\left(F_{\mathrm{d}}=10.64, P=0.022 ; F_{\text {interaction }}=6.35, P<0.0001\right)$, day 1 vs. day 4 $\left(F_{\mathrm{d}}=13.5, P=0.014, F_{\text {interaction }}=6.93, P<0.0001\right)$ and day 2 vs. day $4\left(F_{\mathrm{d}}=16.26, P=0.01, F_{\text {interaction }}=2.92, P=0.006\right)$. The saline pretreated group also differed significantly from the "day 1: AMPH" group, $F_{\text {interaction }}=4.42, P<0.0001$ ). HVA: essentially the same pattern of group differences as seen for DOPAC were found for HVA (statistics not presented). 5-HIAA: the overall 2-way ANOVA with repeated measures on both factors did not result in a significant effect of day of dialysis $\left(F_{\mathrm{d}}=0.75, P=0.5\right)$ or a day $\mathrm{X}$ interval interaction $\left(F_{\text {interaction }}=1.46, P=0.12\right)$. The effect of interval was significant $\left(F_{\mathrm{i}}=9.54, P<0.0001\right)$, indicating that there was a small increase in 5-HIAA after amphetamine administration in all groups. The saline pretreated group did not differ from the amphetamine pretreated group at any time. 
4 days of dialysis $(F=1.69, P=0.19$, within-subjects ANOVA), and only a very small decrease in the basal concentration of 5-HIAA in dialysate, which was not quite statistically significant $(F=$ 2.61, $P=0.07$; although Fisher's tests resulted in a significant difference between the day 4 value and the values obtained on days 1 and $2, P<0.05$; Fig. 1).

Fig. 2 illustrates the time course of the effect of amphetamine on the concentrations of DA, DOPAC, HVA and 5-HIAA on days 1, 2 and 4 of dialysis in those animals that received daily amphetamine $(n=6)$, and on day 4 of testing in saline pretreated animals $(n=5)$. On day 1 , amphetamine produced a large increase in the concentration of DA in dialysate, as expected (Robinson and Camp, 1990). However, responsiveness to amphetamine was greatly attenuated on all subsequent days of testing (overall 2-way ANOVA with repeated measures on both factors, day and interval, $F_{\mathrm{d}}$ (effect of day) $=19.8, P<$ $0.0001, F_{\mathrm{i}}$ (day by interval interaction) $=7.05, P$ $<0.0001$ ). Fig. $2 \mathrm{~A}$ shows that the effect of amphetamine on dialysate DA was significantly reduced on both day 2 ( $F_{\mathrm{d}}=11.67, P=0.019 ; F_{\mathrm{i}}=$ 5.71, $P<0.0001)$ and day $4\left(F_{\mathrm{d}}=24.4, P=0.004\right.$; $\left.F_{\mathrm{i}}=12.13, P<0.0001\right)$, relative to day 1 . The day 4 values were also significantly less than the day 2 values $\left(F_{\mathrm{d}}=20.37, P=0.006 ; F_{\mathrm{i}}=2.21, P=0.03\right)$. Furthermore, when saline pretreated rats received amphetamine for the first time on day 4 they showed a significantly smaller increase in DA than did those animals receiving amphetamine for the first time on day $1\left(F_{\mathrm{i}}=3.2\right.$, $P<0.002$ ), but did not differ from the amphetamine pretreated group when the latter were tested on either days 2 or 4 .

Fig. 2B,C shows the typical amphetamine-induced decrease in the dialysate concentrations of DOPAC and HVA, respectively. The progressive decline in basal DOPAC and HVA across the 4 days of testing is clearly evident, as is the absence of any marked change in 5-HIAA (Fig. 2D).

It is interesting that the magnitude of the decline in amphetamine-stimulated DA release over the 4 days of testing was predicted by the magnitude of the decrease in basal DOPAC but was not related to changes in basal DA. This is illustrated by correlational analyses of the change in basal DOPAC, basal DA, and peak amphetamine-stimulated DA release between days 1 and 4. The percent change in basal DOPAC and peak amphetamine-stimulated DA release between days 1 and 2, and between days 1 and 4, were strongly correlated $(r=+0.835$ and +0.813 , $P<0.01$, respectively). However, there was no significant correlation between the change in either basal DOPAC and basal DA (between days 1 and $4, r=+0.247$ ) or basal DA and peak amphetamine-stimulated DA release (between days 1 and $4, r=+0.311$ ).

\section{Experiment 2}

Fig. 3 shows the average basal concentrations of DA, DOPAC, HVA and 5-HIAA on each of the 4 days of dialysis in Exp. 2. There was no difference between cocaine $(n=5)$ and saline $(n=4)$ pretreated animals in the basal concentration of DA $(F=0.25, P=0.63)$, DOPAC $(F=$ $0.69, P=0.43)$, HVA $(F=2.29, P=0.17)$ or 5 HIAA $(F=0.35, P=0.57)$ across the 4 days of testing (2-way ANOVAs), and therefore, these groups were pooled to simplify data presentation and analysis in Fig. 3.

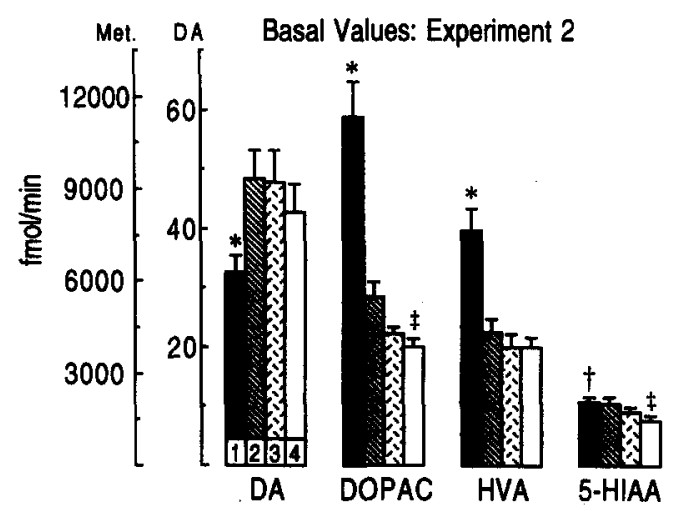

Fig. 3. The mean (+SEM) basal concentrations of DA, DOPAC, HVA, and 5-HIAA in dialysate on each of 4 consecutive days of continuous dialysis in Exp. 2 (days 1-4 are represented as described in Fig. 1). Note that there is a different vertical axis for DA and for the 3 monoamine metabolites (Met). For each compound: *, indicates day 1 values that differed significantly from all other days; $\dagger$, indicates day 4 values that differed from day 2 values; $\ddagger$, indicates that this day 1 value differed only from the day 3 and day 4 values. $(n=9)$. See the text for presentation of statistics. 
Fig. 3 shows that in Exp. 2 there was a marked decrease in the basal concentrations of DOPAC and HVA in dialysate across the 4 days of testing (for DOPAC, $F=43.5, P<0.0001$; for HVA, $F=29.4, P<0.0001)$. For DOPAC, there was a large decrease between days 1 and 2 (Fisher's LSD test, $P<0.05$ ), and a further significant decrease between days 2 and $4(P<0.05)$. The day 3 value was intermediate. For HVA there was also a large decrease between days 1 and 2 $(P<0.05)$, and this appeared to be maximal (i.e., there was no difference between days 2,3 and 4; see Fig. 3). In contrast, there was no decrease in the basal concentration of DA across the 4 days

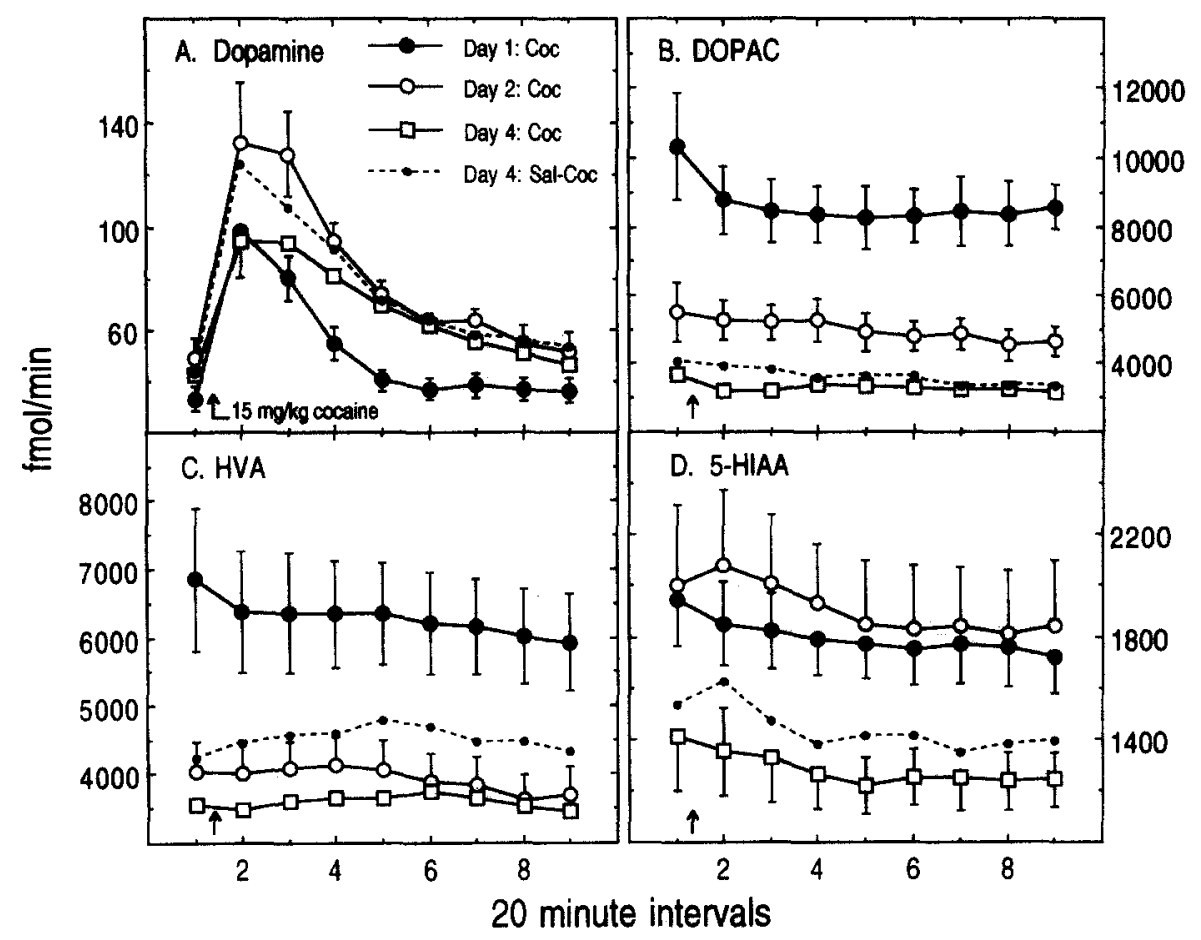

Fig. 4. The effect of $15 \mathrm{mg} / \mathrm{kg}$ of cocaine ("Coc") on the mean ( \pm SEM) concentration of DA, DOPAC, HVA and 5-HIAA in dialysate on days 1, 2 and 4 after probe implantation ("Coc" groups). The "day 4: Sal-Coc" group represents animals that received saline on the first 3 days of dialysis, and their response to cocaine on day 4 of dialysis is shown here. The first 20 min interval represents the basal concentration of each compound (mean of 3 determinations), and each subsequent 20 min interval represents successive samples obtained beginning immediately after the injection of cocaine (indicated by the arrows). Dopamine: see text for presentation of statistics. Briefly, the day 1 values were significantly different from both the day 2 and the day 4 values, and the "day 4: Sal-Coc" group did not differ significantly from the "Coc" group on any day of dialysis. DOPAC: an overall 2-way ANOVA with repeated measures on both factors (day and interval) was significant $\left(F_{\mathrm{d}}=35.1, P<0.0001 ; F_{\mathrm{i}}=2.54, P=0.029 ; F_{\text {interaction }}=\right.$ 2.46, $P=0.006)$, indicating that the extracellular concentration of DOPAC decreased over days of dialysis. Subsequent 2-way ANOVAs showed that there were significant differences between day 1 vs. day $2\left(F_{\mathrm{d}}=20.13, P=0.01 ; F_{\text {interacion }}=2.45\right.$, $P \neq 0.034)$, day 1 vs. day $4\left(F_{\mathrm{d}}=60.4, P<0.001 ; F_{\text {interaction }}=3.4, P=0.006\right)$, and day 2 vs. day $4\left(F_{\mathrm{d}}=20.6, P=0.01 ; F_{\text {interaction }}=\right.$ $1.15, P=0.36)$. The saline pretreated animals also differed significantly from the "day $1:$ Coc" group $\left(F_{\mathrm{d}}=22.7, P=0.002\right.$, $F_{\text {interaction }}=1.34, P=0.24$ ), but other comparisons were not significant. HVA: essentially the same pattern of group differences as seen for DOPAC were found for HVA, except, the day 2 vs. day 4 comparison was not significant, and the saline pretreated animals did not differ from any other group (statistics not shown). 5-HIAA: an overall ANOVA with repeated measures on both factors was significant $\left(F_{\mathrm{d}}=10.97, P=0.005 ; F_{\mathrm{i}}=1.29, P=0.23 ; F_{\text {interaction }}=4.85, P=0.001\right)$, indicating that 5-HIAA changed over days of dialysis. Subsequent 2-way ANOVAs showed that there were no significant differences between day 1 and day 2 $\left(F_{\mathrm{d}}=0.5 ; F_{\text {interaction }}=1.43, P=0.22\right)$, but day 1 did differ from day $4\left(F_{\mathrm{d}}=45.5, P=0.003\right)$, and day 2 differed from day 4 $\left(F_{\mathrm{d}}=13.36, P=0.022\right)$. The "day 4 : Sal-Coc" group did not differ significantly from any other group. 
of dialysis (Fig. 3), and in fact, basal DA was significantly higher on days 2,3 and 4 than on day $1(F=4.88, P=0.009$; follow-up Fisher's LSD tests, $P<0.05$ ). Relative to the DA metabolites, there was only a small change in the basal concentration of 5-HIAA across the 4 days of testing, although in contrast to Exp. 1, it was statistically significant in this experiment $(F=8.67, P=$ $0.0004)$. By day 4, 5-HIAA was significantly lower than on days 1 or 2 , and the day 3 value was significantly lower than the day 1 value $(P<0.05)$. Note, however, as in Exp. 1, there was no difference in 5-HIAA between days 1 and 2, which is when the most marked decrease in the DA metabolites occurred (Fig. 3).

Fig. 4 illustrates the time course of the effect of cocaine on the concentrations of DA, DOPAC, HVA and 5-HIAA in dialysate on days 1, 2 and 4, in those animals that received daily cocaine, and on day 4 of testing in the saline pretreated animals. Cocaine significantly enhanced the concentration of DA in dialysate on all days of testing, although the effect of cocaine changed over the 4 days of testing (2-way ANOVA with repeated measures on both factors, day and time, $F_{\mathrm{d}}=$ 10.71, $P=0.005 ; F_{\mathrm{i}}=1.95, P=0.032$ ). Fig. 4 A shows that, in contrast to the decrease in the effectiveness of amphetamine seen in Exp. 1, there appeared to be a significant increase in the effectiveness of cocaine over days of dialysis in this experiment. The day 2 values were significantly greater than the day 1 values $\left(F_{\mathrm{d}}=13.31\right.$, $P=0.022$ ), and the day 4 values were significantly different than the day 1 and the day 2 values (day 1 vs. day $4: F_{\mathrm{d}}=16.95, P=0.015$; day 2 vs. 4 : $F_{\mathrm{i}}=4.83, P=0.001$ ). Cocaine also significantly elevated DA in saline pretreated rats, but the response to cocaine in this group did not differ significantly from that in cocaine pretreated animals on any day of testing (2-way ANOVAs).

The apparent increase in the effectiveness of cocaine in elevating DA in dialysate (Fig. 4A) should be interpreted with caution, however, because (1) the basal concentration of DA also increased over the 4 days of testing, and (2) the cocaine pretreated group did not differ from the saline pretreated group (Fig. 3). Thus, if the cocaine-induced elevation in DA is expressed as a percent of baseline (data not shown) there is no statistically significant difference between the 4 groups illustrated in Fig. 4A at any single point in time. Nevertheless, even when DA is expressed as a percent of baseline, there was clearly no decrease in the effect of cocaine over 4 days of continuous dialysis, as was the case for amphetamine-stimulated DA release (compare Figs. 2 and 4$)$.

Fig. 4B,C further illustrates the marked decline in the basal concentration of DOPAC and HVA over the 4 days of dialysis (for DOPAC, $F_{\mathrm{d}}=35.1, P<0.0001$; for HVA, $F_{\mathrm{d}}=8.49, P=$ 0.011 ), and the modest change in 5-HIAA (Fig. 4D). It can also be seen in Fig. 4C that acute cocaine administration did not produce the marked reduction in DA metabolites produced by amphetamine.

\section{Discussion}

Previous studies on the effects of continuous dialysis in the nigrostriatal DA system give the impression that this procedure is associated with a parallel decrease in the basal levels of both DA and DA metabolites in dialysate and in amphetamine-stimulated DA release (Imperato and Di Chiara, 1985; Westerink and Tuinte, 1986). In the present study, however, the marked decline in DA metabolites and amphetamine-stimulated DA release seen over 4 days of continuous dialysis was not accompanied by a significant decrease in basal DA (also compare groups 2 and 4 in Table I of Osborne et al. (1990)). In addition, it was found that the magnitude of the decrease in amphetamine-stimulated DA release was predicted by the magnitude of the decrease in basal DOPAC, but there was no significant correlation between the change in amphetamine-stimulated DA release and the change in basal DA. Thus, the present study establishes that the decrease in DA metabolites and amphetamine-stimulated DA release associated with a few days of continuous dialysis is not necessarily accompanied by comparable changes in basal DA.

The decrease in the concentration of compounds in dialysate associated with continuous 
dialysis has usually been attributed to a decline in "recovery", secondary to gliosis-related changes in diffusion (Westerink and Tuinte, 1986; Benveniste and Diemer, 1987). It is unlikely, however, that a relatively non-specific decline in "recovery" caused by a glial "barrier" can account for the pattern of changes reported here, because this would be expected to influence basal DA, as well as DA metabolites and amphetamine-stimulated DA release. Furthermore, the difference between DA and its metabolites is probably not related to the fact that the latter are acids, because in neither experiment reported here was there a change in basal 5-HIAA between days 1 and 2 of testing, which is when basal DOPAC and HVA showed the greatest decline (also see Reiriz et al., 1989). Had the present experiment extended beyond 4 days, however, recovery of DA may have decreased due to the development of a glial "barrier", as suggested previously (Westerink and Tuinte, 1986; Benveniste and Diemer, 1987). This effect may have been seen soon after implantation of a dialysis probe in previous experiments because of a variety of methodological differences, including the size and type of dialysis probe, the perfusion medium and details of the experimental protocol.

If the decrease in dialysate levels of DA metabolites and amphetamine-stimulated DA release was not due to impaired diffusion, what did cause these effects? One possibility is that these measures reflect the integrity of the dopaminergic innervation to the striatum and, thus, the extent to which probe implantation damaged DA terminals. It follows, of course, that the basal concentration of DOPAC in dialysate provides a much better indicator of the integrity of the nigrostriatal DA system than does the basal concentration of DA. In fact, basal DA may be a particularly unreliable indicator of the integrity of the dopaminergic input to the striatum. This interpretation is supported by recent studies on the effects of striatal DA depletion induced by 6-hydroxydopamine or methamphetamine on the concentrations of DA and DA metabolites in dialysate (Robinson and Whishaw, 1988; Touchet and Bennett, 1989; Abercrombie et al., 1990; Castañeda et al., 1990; Robinson et al., 1990).
These latter studies show that the basal concentration of DA in dialysate does not reflect the extent of damage to the nigrostriatal DA system until $\sim 90-95 \%$ of the DA input to the striatum is destroyed, presumably because a number of compensatory neural adaptations to partial injury interact to normalize the basal extracellular concentration of DA (Robinson et al., 1990; Zigmond et al., 1990). On the other hand, the basal concentration of DOPAC in dialysate does accurately reflect the degree of denervation (Abercrombie et al., 1990; Castañeda et al., 1990; Robinson et al., 1990 for review).

Amphetamine is a potential neurotoxin, and high doses can produce a very long-lasting depletion in the postmortem tissue concentration of striatal DA due to the degeneration of DA terminals in the striatum (Ellison et al., 1978; Seiden and Ricaurte, 1987; Ryan et al., 1990). It is important to consider, therefore, whether hypothesized damage to the nigrostriatal DA system reflected by the decrease in amphetaminestimulated DA release and DA metabolites seen on days 2, 3 and 4 after probe implantation could be due to exposure to amphetamine during the first test session. This is unlikely for a number of reasons. First, the dose of amphetamine used here was far below that usually required for amphetamine neurotoxicity (Robinson and Becker, 1986). This is not a strong argument, however, because it is possible that tissue already damaged by probe implantation is more susceptible to the neurotoxic effects of amphetamine (e.g., Phebus et al., 1991). It is important, therefore, that saline pretreated animals also showed a decrease in amphetamine-stimulated DA release, relative to animals that received amphetamine during the first test session and did not differ from amphetamine pretreated animals tested on day 4 . This is strong support for the idea that it was probe implantation, and not prior experience with amphetamine, that was responsible for the deficit. Lastly, a number of microdialysis studies using between-subjects designs have shown that prior amphetamine treatment does not decrease DA release, but increases the DA release induced by a subsequent challenge injection of amphetamine (Ichikawa, 1988; Robinson et al., 1988; Kazahaya 
et al., 1989; Patrick et al., 1991). It is most probable, therefore, that the decrease in amphetamine-stimulated DA release seen over 4 days of continuous dialysis is primarily a consequence of probe-induced alterations in the integrity of the nigrostriatal DA system, and does not reflect the influence of prior amphetamine treatment on DA neurotransmission.

Another finding that deserves discussion concerns the difference in the effect of probe implantation on the ability of cocaine vs. amphetamine to increase the concentration of DA in dialysate. Amphetamine-stimulated DA release was progressively and markedly decreased over the 4 days of testing, but there was no decrease in the ability of cocaine to elevate DA over this same period of time. Unfortunately, it is difficult to directly compare these 2 experiments because a different perfusion medium was used in each (they were conducted 3 years apart). For example, the higher basal level of DA in Exp. 1 is probably because the perfusion medium used in Exp. 1 had a higher concentration of $\mathrm{Ca}^{2+}$ than that used in Exp. 2 (Westerink et al., 1988; Moghaddam and Bunney, 1989). However, amphetamine and cocaine also have a very different mechanism of action, and there is reason to believe this may be the critical variable.

Cocaine acts primarily by binding to the DA transporter, preventing the reuptake of DA, but has little or no effect on DA release (Kuczenski, 1983; Nicolaysen and Justice, 1988). Therefore, the accumulation of extracellular DA seen following cocaine is thought to originate from a vesicular, readily releasable pool of DA by a $\mathrm{Ca}^{2+}$-dependent release process (Hurd and Ungerstedt, 1989) which is presumably due to the spontaneous discharge of DA neurons. Although cocaine can inhibit the discharge of DA neurons by hyperpolarization, this action of cocaine seems to require a higher brain concentration of cocaine than is necessary to block reuptake and affect synaptic activity (Lacey et al., 1990; Uchimura and North, 1990). Thus, the elevation in extracellular DA produced by cocaine may be viewed simply as an accumulation of "basal" DA (i.e., that due to spontaneous release), due to reuptake blockade. Given that there was no decrease in basal DA over the 4 days of dialysis and that cocaine simply produces an accumulation of basal DA, it is not surprising that the effect of cocaine did not change over this period of time either.

Amphetamine, on the other hand, is a potent DA "releaser" (Kuczenski, 1983). Amphetamine is thought to release DA from a cytoplasmic pool of unbound DA by a $\mathrm{Ca}^{2+}$-independent, exchangediffusion process that requires ongoing DA synthesis (Fischer and Cho, 1979; Kuczenski, 1983; Justice et al., 1988), and this occurs independent of neuronal discharge and autoreceptor regulation (Kamal et al., 1981; Kuczenski et al., 1990). The marked decline in amphetamine-stimulated DA release seen between 2 and 4 days after probe implantation is presumably related, therefore, to a disruption of some aspect(s) of this release process. The experiments reported here do not allow us to conclude, however, whether the decline in stimulated DA release is unique to amphetamine-stimulated DA release. It may not be, because Westerink and Tuinte (1986) reported that $\mathrm{K}^{+}$-stimulated DA release also decreases between the day after probe implantation and the next day (although they used a relatively large U-shaped probe). Nevertheless, it appears that either following 2-4 days of chronic probe implantation, or following a second probe insertion at the same site (Camp and Robinson, 1991), DA systems are able to maintain basal extracellular levels of DA but not the demands for increased DA release imposed by an amphetamine challenge. The deficit in stimulated DA release may involve an inability to maintain DA synthesis, or some other aspect(s) of the biochemical cascade required for sustaining large amounts of releasable DA; and changes in other aspects of neurotransmission cannot be excluded at this time. It is certainly possible that complex interactions between many processes are involved, and determination of the exact nature of the deficit will require further experimentation.

Whatever the reason for the deficit in amphetamine-stimulated DA release reported here, the results suggest that within-subjects design experiments involving continuous dialysis over a few days in DA systems probably should be avoided. The effects of repeated probe insertions at the 
same site are very similar to those reported here, suggesting that this approach may not be appropriate either (Camp and Robinson, 1991; Robinson and Camp, 1991). Whether within-subjects designs are feasible for studying other neurotransmitter systems, or animals with a larger brain (e.g., Kendrick, 1991), remain open questions. For example, the basal concentrations of aspartate, threonine and glutamate in dialysate from the substantia nigra of cats are reported to be stable over ten months (Delgado et al., 1984). On the other hand, Korf and Venema (1985) have reported that although some amino acids are stable over 9 days of dialysis, others are not, and the response to $\mathrm{K}^{+}$changes over 9 days of dialysis. Of course, there is the question of the source of amino acids sampled with dialysis in brain, and it has been suggested that a significant proportion may not be of neural origin (Westerink and de Vries, 1989; Westerink et al., 1987, 1988, 1989).

In conclusion, the experiments reported here suggest that with the technology presently available within-subjects design studies involving continuous microdialysis in the DA system of small animals are ill advised. If microdialysis is used for within-subjects design experiments, it should be incumbent upon the investigator to demonstrate that the integrity of the system under study is maintained between test sessions. Most importantly, the data presented here establish that a "stable" concentration of a neurotransmitter in dialysate does not constitute such evidence, and in fact, this may be the least sensitive indicator of the integrity of a neurotransmitter system (Robinson et al., 1990).

\section{Acknowledgements}

This research was supported by grant no. 04294 from the National Institute on Drug Abuse. We thank Simon Ng and Erin Wolfe for their assistance in conducting the experiments. Some of the data reported here were reported previously in abstracts (Camp and Robinson, 1989, 1990), or in preliminary form in a non-reviewed book chapter (Robinson and Camp, 1991).

\section{References}

Abercrombie, E.D., Bonatz, A.E. and Zigmond, M.J. (1990) Effects of 1-Dopa on extracellular dopamine in striatum of normal and 6-hydroxydopamine-treated rats, Brain Res., 525: $36-44$.

Benveniste, H. and Diemer, N.H. (1987) Cellular reactions to implantation of a microdialysis tube in the rat hippocampus, Acta Neuropathol. (Berl.), 74: 234-238.

Benveniste, H. and Hansen, A.J. (1991) Practical aspects of using microdialysis for determination of brain interstitial concentrations. In: T.E. Robinson and J.B. Justice, Jr. (Eds.), Microdialysis in the Neurosciences, Elsevier, Amsterdam, pp. 81-100.

Benveniste, H. and Hüttemeier, P.C. (1990) Microdialysis theory and application, Prog. Neurobiol., 35: 195-215.

Camp, D.M. and Robinson, T.E. (1989) A method for repeated intracerebral microdialysis, Soc. Neurosci. Abst., 15: 559 .

Camp, D.M. and Robinson, T.E. (1990) Repeated intracerebral microdialysis for the measurement of stimulated dopamine release, Soc. Neurosci. Abst., 16: 130.

Camp, D.M. and Robinson, T.E. (1991) The use of multiple probe insertions at the same site for repeated intracerebral microdialysis experiments in the mesostriatal dopamine system of rats, submitted.

Castañeda, E., Whishaw, I.Q. and Robinson, T.E. (1990) Changes in striatal dopamine neurotransmission assessed with microdialysis following recovery from a bilateral 6OHDA lesion: variation as a function of lesion size, $\mathrm{J}$. Neurosci., 10: 1847-1854.

Delgado, J.M., Lerma, J., Martin del Rio, R. and Solis, J.M. (1984) Dialytrode technology and local profiles of amino acids in the awake cat brain, J. Neurochem., 42: 1218-1228.

Ellison, G., Eison, M.S., Huberman, H.S. and Daniel, F. (1978) Long-term changes in dopaminergic innervation of caudate nucleus after continuous amphetamine administration, Science, 201: 276-278.

Fischer, J.F. and Cho, A.K. (1979) Chemical release of dopamine from striatal homogenates: evidence for an exchange diffusion model, J. Pharmacol. Exp. Ther., 208: 203-209.

Hamberger, A., Berthold, C.H., Karlsson, B., Lehmann, A. and Nyström, B. (1983) Extracellular GABA, glutamate and glutamine in vivo-perfusion dialysis of the rabbit hippocampus. In: L. Herz, E. Kvamme, E.G. McGeer and A. Schousboe (Eds.), Glutamine, Glutamate and GABA in the Central Nervous System, Alan R. Liss, New York, pp. 473-492.

Hurd, Y.L. and Ungerstedt, U. (1989) Cocaine: an in vivo microdialysis evaluation of its acute action on dopamine transmission in rat striatum, Synapse, 3: 48-54.

Ichikawa, J. (1988) Changes in behavior and central monoaminergic systems in the rat after repeated methamphetamine pretreatment: presynaptic regulatory mechanism, Yakubutsu Seishin Kodo, 8: 389-403.

Imperato, A. and Di Chiara, G. (1984) Trans-striatal dialysis 
coupled to reverse phase high performance liquid chromatography with electrochemical detection: a new method for the study of the in vivo release of endogenous dopamine and metabolites, J. Neurosci., 4: 966-977.

Imperato, A. and Di Chiara, G. (1985) Dopamine release and metabolism in awake rats after systemic neuroleptics as studied by trans-striatal dialysis, J. Neurosci., 5: 297-306.

Justice, J.J., Nicolaysen, L.C. and Michael, A.C. (1988) Modeling the dopaminergic nerve terminal, J. Neurosci. Methods, 22: 239-252.

Kamal, L.A., Arbilla, S. and Langer, S.Z. (1981) Presynaptic modulation of the release of dopamine from the rabbit caudate nucleus: differences between electrical stimulation, amphetamine and tyramine, J. Pharmacol. Exp. Ther., 216: 592-598.

Kazahaya, Y., Akimoto, K. and Otsuki, S. (1989) Subchronic methamphetamine treatment enhances methamphetamine- or cocaine-induced dopamine efflux in vivo, Biol. Psychiat., 25: 903-912.

Kendrick, K.M. (1991) Microdialysis in large unrestrained animals: neuroendocrine and behavioral studies of acetylcholine, amino acid, monoamine and neuropeptide release in the sheep. In: T.E. Robinson and J.B. Justice, Jr. (Eds.), Microdialysis in the Neurosciences, Elsevier, Amsterdam, pp. 327-348.

Korf, J. and Venema, K. (1985) Amino acids in rat striatal dialysates: methodological aspects and changes after electroconvulsive shock, J. Neurochem., 45: 1341-1348.

Kuczenski, R. (1983) Biochemical actions of amphetamine and other stimulants. In: I. Creese (Ed.), Stimulants: Neurochemical, Behavioral and Clinical Perspectives, Raven Press, New York, pp. 31-61.

Kuczenski, R., Segal, D.S. and Manley, L.D. (1990) Apomorphine does not alter amphetamine-induced dopamine release measured in striatal dialysates, J. Neurochem., 54: $1492-1499$

Lacey, M.G., Mercuri, N.B. and North, R.A. (1990) Actions of cocaine on rat dopaminergic neurones in vitro, $\mathrm{Br}$. $\mathrm{J}$. Pharmacol., 99: 731-735.

Moghaddam, B. and Bunney, B.S. (1989) Ionic composition of microdialysis perfusing solution alters the pharmacological responsiveness and basal outflow of striatal dopamine, J. Neurochem., 53: 652-654.

Morrison, P.F., Bungay, P.M., Hsiao, J.K., Mefford, I.N., Dykstra, K.H. and Dedrick, R.L. (1991) Quantitative microdialysis. In: T.E. Robinson and J.B. Justice, Jr. (Eds.), Microdialysis in the Neurosciences, Elsevier, Amsterdam, pp. $47-80$.

Nicolaysen, L.C. and Justice, J.B.J. (1988) Effects of cocaine on release and uptake of dopamine in vivo: differentiation by mathematical modeling, Pharmacol. Biochem. Behav., 31: 327-335.

Osborne, P.G., O'Connor, W.T., Drew, K.L. and Ungerstedt, U. (1990) An in vivo microdialysis characterization of extracellular dopamine and GABA in dorsolateral striatum of awake freely moving and halothane anaesthetised rats, J. Neurosci. Methods, 34: 99-105.
Patrick, S.L., Thompson, T.L., Walker, J.M. and Patrick, R.L. (1991) Concomitant sensitization of amphetamine-induced behavioral stimulation and in vivo dopamine release from the rat caudate nucleus, Brain Res., 538: 343-346.

Phebus, L.A., Mincy, R.E. and Clemens, J.A. (1991) Microdialysis perfusion accelerates striatal ischemic damage, Curr. Sep., 10: 114.

Reiriz, J., Mena, M.A., Bazan, E., Muradas, V., Lerma, J., Delgado, J.M. and De Yebenes, J.G. (1989) Temporal profile of levels of monoamines and their metabolites in striata of rats implanted with dialysis tubes, J. Neurochem. 53: 789-792.

Robinson, T.E. and Becker, J.B. (1986) Enduring changes in brain and behavior produced by chronic amphetamine administration: a review and evaluation of animal models of amphetamine psychosis, Brain Res. Rev., 396: 157-198.

Robinson, T.E. and Camp, D.M. (1990) Does amphetamine preferentially increase the extracellular concentration of dopamine in the mesolimbic system of freely moving rats?, Neuropsychopharmacology, 3: 163-173.

Robinson, T.E. and Camp, D.M. (1991) The feasibility of repeated microdialysis for within-subjects design experiments: studies on the mesostriatal dopamine system. In: T.E. Robinson and J.B. Justice, Jr. (Eds.), Microdialysis in the Neurosciences, Elsevier, Amsterdam, pp. 189-234.

Robinson, T.E. and Whishaw, I.Q. (1988) Normalization of extracellular dopamine in striatum following recovery from a partial unilateral 6-OHDA lesion of the substantia nigra: a microdialysis study in freely moving rat, Brain Res., 450 209-224.

Robinson, T.E., Jurson, P.A., Bennett, J.A. and Bentgen, K.M. (1988) Persistent sensitization of dopamine neurotransmission in ventral striatum (nucleus accumbens) produced by past experience with $(+)$-amphetamine: a microdialysis study in freely moving rats, Brain Res., 462: 211222.

Robinson, T.E., Castañeda, E. and Whishaw, I.Q. (1990) Compensatory changes in striatal dopamine neurons following recovery from injury induced by 6-OHDA or methamphetamine: a review of evidence from microdialysis studies, Can. J. Psychol., 44: 253-275.

Ryan, L.J., Linder, J.C., Martone, M.E. and Groves, P.M. (1990) Histological and ultrastructural evidence that $D^{-}$ amphetamine causes degeneration in neostriatum and frontal cortex of rats, Brain Res., 518: 67-77.

Santiago, M. and Westerink, B.H.C. (1990) Characterization of the in vivo release of dopamine as recorded by different types of intracerebral microdialysis probes, Naunyn Schmiedebergs Arch. Pharmacol., 342: 407-414.

Seiden, L.S. and Ricaurte, G.A. (1987) Neurotoxicity of methamphetamine and related drugs. In: H.Y. Melzer (Ed.), Psychopharmacology: The Third Generation of Progress, Raven Press, New York, pp. 359-366.

Touchet, N. and Bennett, J.J. (1989) The metabolism of systemically-administered L-dihydroxyphenylalanine, by intact and dopamine-denervated striata, as revealed by brain microdialysis, Neuropharmacology, 28: 1217-1222. 
Uchimura, N. and North, R.A. (1990) Actions of cocaine on rat nucleus accumbens neurones in vitro, Br. J. Pharmacol., 99: $736-740$.

Ungerstedt, U. and Hallström, A. (1987) In vivo microdialysis - a new approach to the analysis of neurotransmitters in the brain, Life Sci., 41: 861-864.

Westerink, B.H. and De Vries, J.B. (1988) Characterization of in vivo dopamine release as determined by brain microdialysis after acute and subchronic implantations: methodological aspects, J. Neurochem., 51: 683-687.

Westerink, B.H.C. and De Vries, J.B. (1989) On the origin of extracellular GABA collected by brain microdialysis and assayed by a simplified on-line method, Naunyn Schmiedebergs Arch. Pharmacol., 339: 603-607.

Westerink, B.H. and Tuinte, M.H. (1986) Chronic use of intracerebral dialysis for the in vivo measurement of 3,4dihydroxyphenylethylamine and its metabolite 3,4-dihydroxyphenylacetic acid, J. Neurochem., 46: 181-185.
Westerink, B.H., Damsma, G., Rollema, H., De Vries, J.B. and Horn, A.S. (1987) Scope and limitations of in vivo brain dialysis: a comparison of its application to various neurotransmitter systems, Life Sci., 41: 1763-1776.

Westerink, B.H., Hofsteede, H.M., Damsma, G. and De Vries, J.B. (1988) The significance of extracellular calcium for the release of dopamine, acetylcholine and amino acids in conscious rats, evaluated by brain microdialysis, Naunyn Schmiedebergs Arch. Pharmacol, 337: 373-378.

Westerink, B.H.C., Damsma, G. and De Vries, J.B. (1989) Effect of ouabain applied by intrastriatal microdialysis on the in vivo release of dopamine, acetylcholine and amino acids in the brain of conscious rats, J. Neurochem., 52: 705-712.

Zigmond, M.J., Abercrombie, E.D., Berger, T.W., Grace, A.A., and Stricker, E.M. (1990) Compensations after lesions of central dopaminergic neurons: some clinical and basic implications, Trends Neurosci., 13: 290-296. 